\title{
Effect of macroscopic relaxation on residual stress analysis by diffraction methods
}

\author{
J. Repper, ${ }^{1, a)}$ M. Hofmann, ${ }^{1}$ C. Krempaszky, ${ }^{2,3}$ B. Regener, ${ }^{2,3}$ E. Berhuber, ${ }^{4}$ W. Petry, ${ }^{1}$ \\ and E. Werner ${ }^{2,3}$ \\ ${ }^{1}$ Forschungsneutronenquelle Heinz Maier-Leibnitz (FRM II), TU München, Lichtenbergstr. \\ 1,85747 Garching, Germany \\ ${ }^{2}$ Christian-Doppler-Labor für Werkstoffmechanik von Hochleistungslegierungen, TU München, \\ Boltzmannstr. 15, 85747 Garching, Germany \\ ${ }^{3}$ Lehrstuhl für Werkstoffkunde und Werkstoffmechanik, TU München, Boltzmannstr. \\ 15, 85747 Garching, Germany \\ ${ }^{4}$ Böhler Schmiedetechnik GmbH \& Co KG, Mariazeller Straße 25, 8605 Kapfenberg, Austria
}

(Received 16 May 2012; accepted 22 August 2012; published online 21 September 2012)

To know the residual stress state within a component is essential for predicting its service life time. Neutron diffraction is a reliable tool for non-destructive determination of macroscopic residual stresses, even deep inside a component. One standard procedure in neutron residual stress analysis is to cut out small macrostress free coupons from the strained component, which serve as reference samples. Redistribution of intergranular stresses due to relaxation when the coupon is cut out, however, may lead to spurious macroscopic stresses. A quantitative study of the change of intergranular stresses shows that, contrary to conventional thought, even the $\{311\}$ Bragg reflection is affected strongly by this phenomenon. In this case, the use of reference values calculated using boundary conditions of mechanical equilibrium enables to extract reliable macroscopic residual stress values. @ 2012 American Institute of Physics. [http://dx.doi.org/10.1063/1.4752877]

\section{INTRODUCTION}

It is well known that macroscopic residual stresses stored within components influence their service life time. For instance, compressive macroscopic residual stresses near the surface may enhance the life time by inhibiting the propagation of microcracks. ${ }^{1}$ By contrast, tensile macrostresses may lead to a reduction of strength and toughness due to premature crack initiation. ${ }^{2}$

Mechanical methods and diffraction methods are commonly used to determine residual stresses. ${ }^{3}$ Mechanical methods use the distortion during machining (e.g., turning or drilling) as a measure of the macroscopic residual stress state of the component machined. The use of these methods to identify the stress state is destructive and independent of the underlying microstructure of the material investigated. Diffraction methods use the crystal lattice spacing as an internal strain gauge. They are generally non-destructive and sensitive to the crystal phases. The crystal lattice spacing of a non-strained sample serves as reference value to determine elastic strain. Diffraction experiments are based on Bragg's equation, which connects the measured scattering angle with the crystal lattice spacing. In a diffraction experiment, a superposition of macroscopic and microscopic (here intergranular) strains is always measured since they both cause shifts of the scattering angle. ${ }^{4}$ Microscopic stresses depend on the microstructure of the sample and the elastic and plastic anisotropies of the measured crystal. The common approach to minimize or even cancel out the influence of

\footnotetext{
a) Author to whom correspondence should be addressed. Electronic mail: Julia.Repper@PSI.ch. Tel.: +41 56310 5530.Fax: +41 56310 3131. Present address: Paul Scherrer Institute, Materials Science and Simulation, NUM/ASQ, 5232 Villigen PSI, Switzerland.
}

intergranular strains and stresses on the macroscopic residual stress analysis completely is to use (a) crystal planes, which are known to be quite insensitive to intergranular stresses and/or (b) reference samples, which possess the same intergranular stress state as the component of interest. ${ }^{5}$

Following the latter approach, reference samples are often small, macrostress free coupons taken from the strained component of interest using a cutting procedure which does not alter the microstructure of the material. ${ }^{1,5,6}$ Nevertheless, in case of highly plastically predeformed (e.g., during forging) materials with large crystal anisotropy, in which the response to mechanic load differs in different crystallographic directions, it is conceivable that the intergranular stress state is modified significantly in the cutting/extraction process due to the load employed from macroscopic relaxation.

Here, we present a quantitative study of the change of intergranular residual stresses during the extraction of small reference coupons from forged IN718 disks. The residual stress analysis was performed according to the ISO standard. ${ }^{5}$ Changing intergranular strains leads to large spurious stresses. Dimensions of the sample were chosen so that it also was possible to calculate the stress state in the throughthickness direction using boundary restrictions enforced by mechanical equilibrium conditions. The samples were forged, followed by water quenching which ensured (1) large plastic pre-deformation of the disk and (2) stress gradients large enough to induce plastic deformation, when they are relaxed. By using these samples, it could be shown that the relaxation during extraction of the reference coupons changes the intergranular stress state significantly and that, contrary to conventional thought, even the $\{311\}$ Bragg reflection was strongly affected by this phenomenon. To the best of the authors' knowledge, no similar study exists. 


\section{NEUTRON DIFFRACTION}

In neutron diffraction, the determination of residual stresses in components is nondestructive and sensitive to crystal phases. Neutron diffraction further has the advantage of gathering information from deep inside the material due to the high penetration of neutron radiation into matter (for instance in aluminum, measurements of up to $100 \mathrm{~mm}$ in depth can be realized). In diffraction experiments, the interplanar lattice spacing $d_{h k l}$ of crystallographic lattice planes $\{h k l\}$ is related to the scattering angle $\theta_{h k l}$ by Bragg's law

$$
\lambda=2 d_{h k l} \sin \theta_{h k l},
$$

here $\lambda$ is the wavelength of the neutrons.

Stresses in a sample cause strains which result in a variation of the lattice spacing $\Delta d_{h k l}=d_{h k l}-d_{0}$, with $d_{0}$ the unstrained lattice spacing. For a monochromatic neutron beam, the elastic strain $\varepsilon_{h k l}$ can be directly calculated by comparing the scattering angle of a stressed sample $\left(\theta_{h k l}\right)$ and a stress-free reference sample $\left(\theta_{0, h k l}\right)$ using Eq. (1)

$$
\varepsilon_{h k l}=\frac{\Delta d_{h k l}}{d_{0}}=\frac{\sin \theta_{0, h k l}}{\sin \theta_{h k l}}-1 .
$$

In this case, the strain is measured in the direction of the scattering vector $\vec{Q}$, which is the direction bisecting the directions of incident and diffracted neutron beams.

Determination of the reference value $\theta_{0, h k l}$ is one of the most cumbersome tasks in stress analysis using neutron diffraction, and various approaches have been suggested in the literature. ${ }^{1,6}$ The most reliable ones are also listed in the ISO standard for residual stress determination by neutron diffraction. 5

The stress state as well as the strain state is given by a second order tensor. ${ }^{7}$ Complete determination of the stress tensor requires the measurement of at least six directions of the strain tensor. For highly symmetric, homogeneous and isotropic samples, however, measurements in the three principal axes of the system $\left(x_{1}, x_{2}\right.$, and $\left.x_{3}\right)$ are sufficient. The stress in one of these directions (here denoted as $\sigma_{33}$ ) is only dependent on the strains observed in the three principal strain directions $\left(\varepsilon_{11}, \varepsilon_{22}, \varepsilon_{33}\right)$

$$
\sigma_{33}=\frac{E_{h k l}}{1+\nu_{h k l}}\left(\varepsilon_{33}+\frac{\nu_{h k l}}{1-2 \nu_{h k l}}\left(\varepsilon_{11}+\varepsilon_{22}+\varepsilon_{33}\right)\right) .
$$

Because of that, Young's modulus $E_{h k l}$ and Poisson's ratio $\nu_{h k l}$ are the only diffraction elastic constants. A comprehensive overview on residual stress analysis using neutron diffraction can be found in Refs. 1, 3, 4, and 8 .

\section{SAMPLE MATERIAL AND SAMPLE PREPARATION}

An Inconel 718 (IN 718) disk with a thickness of about $18.5 \mathrm{~mm}$ and a radius of $50 \mathrm{~mm}$ was used in this study. IN 718 is a nickel-based superalloy for high temperature applications and withstands high deformation rates. ${ }^{9}$ Nickel possesses a large crystal anisotropy which leads to differences in the mechanical behavior between different crystallographic directions $\{h k l\}$. The disk used was deformed plastically to a large extent by forging at $990{ }^{\circ} \mathrm{C}$ after holding this temperature for $65 \mathrm{~min}$. After that it was water quenched back to room temperature to ensure high macroscopic residual stresses within the disk. The disk was lowered into the quench bath in a cage to assure that it entered the bath upright. The high quench rate suppresses the formation of precipitates within the nickel matrix. High-resolution neutron powder diffraction shows that the alloy can be treated as single phase material (Figure 1) and thus interphase residual stresses were not considered. In forged and quenched materials, it is usually assumed that the chemical composition varies slightly through the thickness of the disk due to diffusion processes during the heat treatment. ${ }^{12,13}$

\section{EXPERIMENTAL SECTION}

\section{A. Experimental procedure}

The radius of the disk was reduced in three steps by wire electrical discharge machining (WEDM). Each reduction step relaxed macroscopic stresses. Neutron diffraction measurements were conducted along the center-axes of the specimens in the parent disk as well as after each reduction step. Radii at which measurements have been performed were: $50 \mathrm{~mm}$ (original state), 20, 10, and $2 \mathrm{~mm}$ (Figures 2 and 3).

\section{B. Experimental details}

The neutron diffraction experiments were carried out at the materials science diffractometer STRESS-SPEC at FRM II, Munich. ${ }^{14}$ Strains were measured in the three principal directions $x_{1}, x_{2}$, and $x_{3}$ using the $\{311\}$ lattice plane (Figures 2 and 3). A wavelength of $(\lambda=1.556 \AA)$ was chosen bringing the \{311\} Bragg reflection to a scattering angle of $2 \theta \approx 91.3^{\circ}$. A gauge volume of $3 \times 3 \times 3 \mathrm{~mm}^{3}$ was used to ensure good

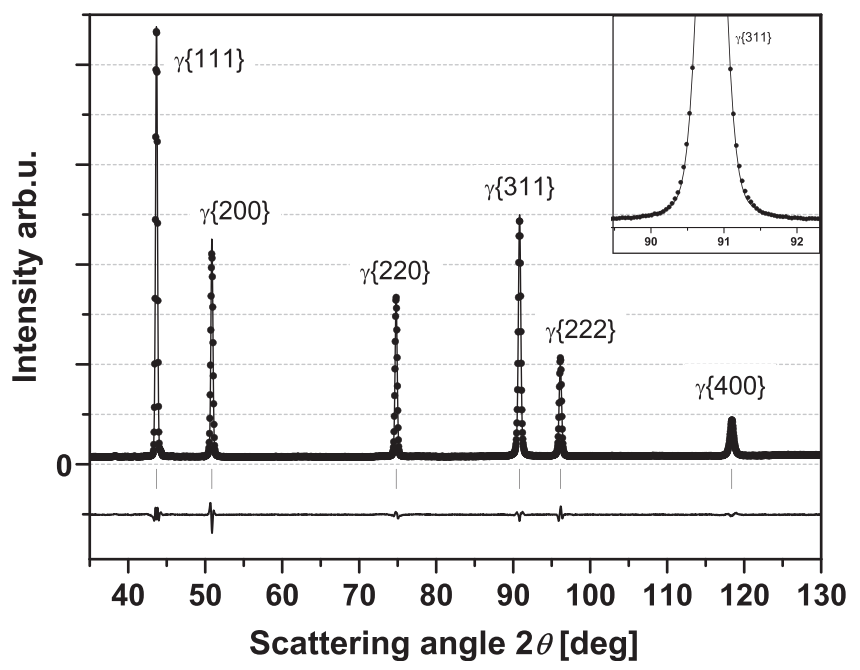

FIG. 1. LeBail refinement (diffraction pattern solid line) ${ }^{10}$ of a neutron diffraction pattern (dots) of the sample with a radius of $2 \mathrm{~mm}$. The measurement with a wavelength of $\lambda=1.548 \AA$ was conducted at the high resolution powder neutron diffractometer SPODI at the neutron source FRM II. ${ }^{11}$ The symmetric peak shape of the $\{311\}$ Bragg reflection (inlet) shows that only Bragg reflections of the $\gamma$-matrix phase are found ( $2 \theta$ positions marked by vertical lines). The bottom curve shows the difference between the refined model and the experimental data. 


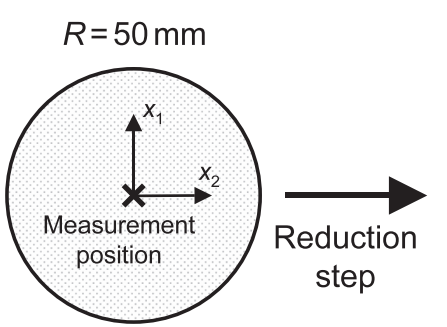

Neutron

measurement
$R=20 \mathrm{~mm} \quad R=10 \mathrm{~mm}$
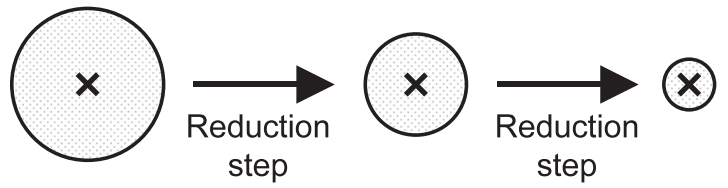

Neutron measurement
Neutron measurement
Neutron measurement
FIG. 2. Schematic sketch of the experimental workflow of the experimental procedure. The radius of the sample was reduced successively by electrical discharge machining. The residual stress distribution across the thickness was determined for all radii by neutron diffraction along the center axis of the sample (cross). The principal axes $x_{1}$ and $x_{2}$ are depicted by arrows for the $R=50 \mathrm{~mm}$ sample. For clarity, the normal direction $x_{3}$ is not indicated. counting statistics in an acceptable time. Through-thickness scans were performed in 11 equidistant steps along the centeraxes symmetrically to the mid-planes of the samples with radii of 50, 20,10, and $2 \mathrm{~mm}$. The step size was $1.5 \mathrm{~mm}$.

Diffraction elastic constants for the $\{311\}$ Bragg reflection $E_{\{311\}}=208.29 \mathrm{GPa}$ and $\nu_{\{311\}}=0.31$ were calculated according to Kröner's law using single crystal nickel data. ${ }^{15}$

\section{ISO STANDARD CONFORMITY}

The residual stress analysis was carried out using an experimental procedure suggested in the ISO standard $21432 .{ }^{5}$

- Concerning the choice of a suitable Bragg reflection, the ISO standard states that "it is important, for engineering residual strain measurements, that a crystallographic plane is chosen which gives essentially zero residual strain on unloading." In the case of Ni-based superalloys, the $\{311\}$ Bragg reflection is known to facilitate this demand. 5,16,17

- Further, "Lattice spacings are sensitive to a number of causes... The most important of these are chemical composition and temperature."

Chemical composition: The lattice spacing variation due to slightly varying chemical composition at different measurement positions was overcome using reference values determined for each measurement position independently. With this approach, the influence of chemical composition and other microstructural inhomogehneities (e.g., degree of plastic deformation) cancels out. ${ }^{12,13}$

Temperature: The temperature in the experimental hall of FRM II was stable within $\pm 0.5^{\circ} \mathrm{C}$ during one measurement (averaged measurement time per point $\approx 25 \mathrm{~min}$ ). This fluctuation causes errors of $\pm 1.5 \mathrm{MPa}\left(\alpha_{\mathrm{IN} 718} \approx 13 \times 10^{-6} /{ }^{\circ} \mathrm{C}\right)$. This error was added to the statistical error found for the gaussian distribution fitted to the data.

- Reference value: The ISO standard suggests to calculate " $d_{0}$ by imposing force and moment equilibrium" or to measure $d_{0}$ "on small coupons, cut from large blocks of material." Both methods were applied in this experiment (see Sec. VIII). In this respect, the ISO standard further suggests: "Care shall be taken with the preparation of "stressfree" material to avoid the introduction of residual stresses, or modification of the microstructure, during manufacture." Therefore, WEDM was used for the reduction of the diameter of the disk. This method is known for its negligible influence on sample microstructure. Neither changes of microstructure nor residual stress fields introduced by the method itself exceed surface depths of more then $0.1 \mathrm{~mm}$, which is irrelevant on the length scale of neutron diffraction experiments (gauge volume: $3 \times 3 \times 3 \mathrm{~mm}^{3}$ ). ${ }^{18,19}$

\section{MECHANICAL EQUILIBRIUM}

Seen from its center region, the disk can be treated as an infinite plate of thickness $h$ due to its sample geometry and the homogeneous thermal boundary conditions during quenching. For infinite plates exhibiting axial symmetryand therefore an isotropic in-plane stress state-the stress in $x_{3}$-direction (axial direction) must vanish.

The residual stress distribution in infinite plates is independent of the in-plane coordinates $x_{1}, x_{2}$, i.e., $\sigma_{i j}=\sigma_{i j}\left(x_{3}\right)$, where $i, j=1,2,3$. Neglecting volume forces, $f_{j}=0$, the mechanical equilibrium $\left(\sum_{i=1}^{n} \sigma_{i j, i}+f_{j}=0\right)$ enforces $\sigma_{3 j, 3}=0$, with $n=3$. Taking into account the mechanical boundary conditions of the free surface, $\sigma_{3 j}$ (at $x_{3}= \pm h / 2=0$ ) yields $\sigma_{3 j}=0$. However, the assumption of an infinite disk is only applicable to disk positions where effects of free radial edges can be neglected. ${ }^{20}$ How free radial edges influence the residual stress state at the center-axis of disk-shaped components depends on the the radius $\mathrm{R}$ to thickness $H$ ratio. $^{21}$ For large $R / H$ ratios, the center of the disk can be treated as an infinite plate, whereas for small $R / H$ ratios (approx. $<1.5$ ) the effect of free edges becomes significant. The ratio $R / H$ is

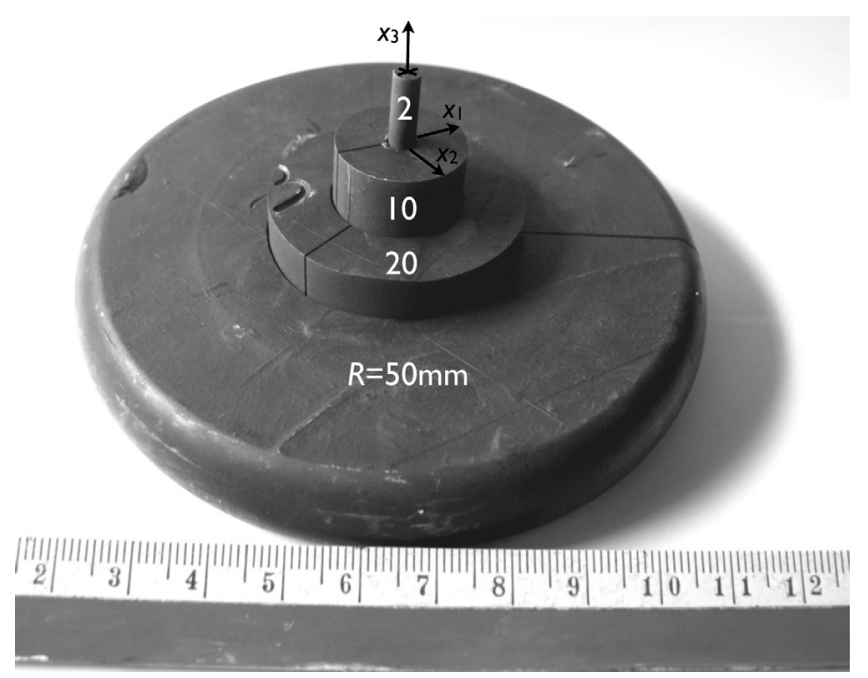

FIG. 3. Photograph of the disk successive staggered from the samples with different radii as cut during the experiment $(R=50,20,10$, and $2 \mathrm{~mm})$. The measurement position was for all radii in the center of the sample (black cross). The principal axes (which are the measurement directions) $x_{1}, x_{2}$, and $x_{3}$ are depicted by black arrows. 
2.7 for the disk with $R=50 \mathrm{~mm}(H=18.5 \mathrm{~mm})$. Therefore, the original disk $(R=50 \mathrm{~mm})$ must exhibit vanishing macroscopic axial stresses $\sigma_{33}$ along its center-axis ( $x_{3}$-direction).

\section{MECHANICAL MODELING}

Finite element modeling (FEM) was used to simulate the ambient temperature macroscopic residual stress state in the disk after quenching as well as the redistribution of the macroscopic stresses after each reduction step by WEDM. Due to the low yield strength of the material at forging temperature, the residual stresses prior to quenching are small and can be neglected. The forging and quenching processes are assumed to be axisymmetric as well as symmetric with respect to the mid-plane of the disk. Therefore, the numerical simulation can be based on an axisymmetric formulation employing fully coupled thermomechanical finite elements. Temperature dependence of Poisson's ratio, Young's modulus, yield stress, density, and thermal diffusivity was taken into account. By contrast, the heat transfer coefficient $\alpha=2400 \mathrm{~W} / \mathrm{Km}^{2}$ is assumed to be independent of temperature and constant over the whole surface of the sample. The model was implemented into the commercial finite element code ABAQUS FEA. All cutting steps are modeled as pure material removal. Simulation results were evaluated at the symmetry axis of the disk.

\section{RESULTS AND DISCUSSION}

Figure 4 shows the through thickness macroscopic residual stress distribution along the center-axis of the parent disk. The $2 \theta$ values measured at the sample with a radius of $2 \mathrm{~mm}$ at identical measurement positions as used for the parent disk, served as reference values. Because of its small dimensions, the sample with $R=2 \mathrm{~mm}$ is stress-free (macroscopically), as verified by finite element simulations (Figure 5). As mentioned before, using this reference sample cancels out the effects of chemical composition variations, as the used measurement positions are identical in reference and

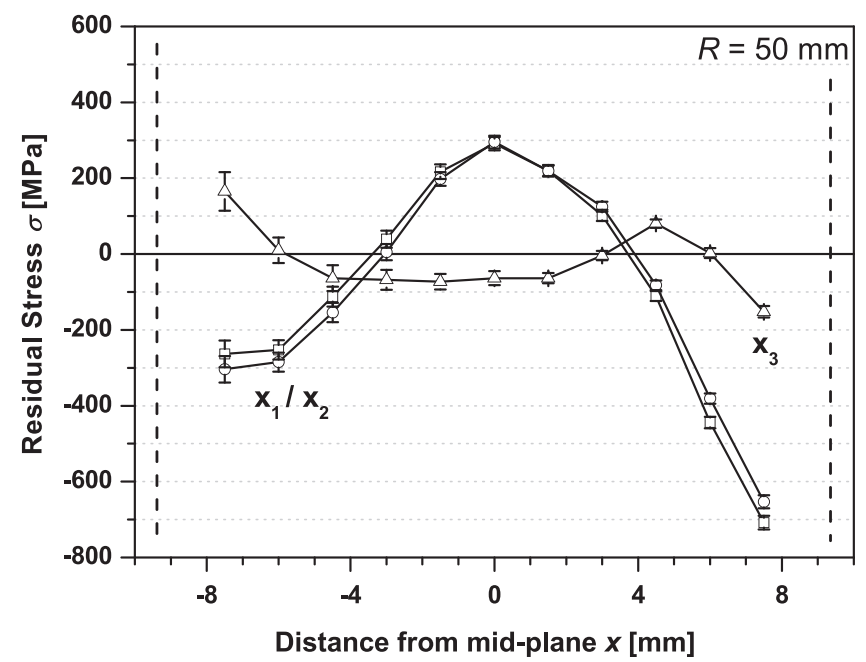

FIG. 4. Residual stress distributions along the through-thickness disk axis of the sample with $R=50 \mathrm{~mm}$ taking the eleven $2 \theta$ values measured at the sample with a radius of $2 \mathrm{~mm}$ as reference values. The dashed lines on both sides of the graphs mark the upper and bottom surfaces of the disk. The residual stresses are given for the principal directions $x_{1}$ (squares), $x_{2}$ (circles), and $x_{3}$ (triangles). original disk. The residual stress distribution in the parent disk shows expected features: compressive stresses at the surfaces of the disk balanced by tensile stresses in its core are expected by the thermal gradient through the thickness of the disk during water quenching (skin-core effect).

The fact that the residual macrostress distributions in $x_{1}$ and $x_{2}$ directions are identical is enforced by the radial symmetry of the sample and the measurement positions chosen along its center-axis (Figure 4). Beside these expected features, Figure 4 also shows clear deviations from the expected trends: First, the experimentally determined stress distribution shows a marked asymmetry. This is surprising as the stresses in all principal directions should be symmetric to the mid-plane of the disk as the forging and quenching processes are assumed to be symmetrical to the mid-plane of the disk. Further, one finds a non zero axial stress component, whereas mechanical equilibrium requires vanishing macroscopic stresses in $x_{3}$-direction as the $R / H$ ratio of the disk is $>1.5$ (see Sec. VI).

The measurement procedure adhered to the ISO standard which excludes the following reasons for this discrepancy: (1) microstructural changes by the cutting process itself as WEDM has only little influence on the sample and (2) microstructural inhomogeneities in the original microstructure (due to the use of the same measurement positions for stressed and stress-free samples). However, the discrepancy can be explained by differences in the intergranular stress state between the original disk $(R=50 \mathrm{~mm})$ and the reference sample $(R=2 \mathrm{~mm})$ as a result of the cutting process. The cutting process relieves load large enough to induce local (secondary) plastic deformation in the sample (further termed here as macroscopic unloading). This is possible as the total stresses of about $800 \mathrm{MPa}$ in the parent disk are of the same order as the yield strength of the material. Then, full, macroscopic unloading may result in elastic straining (e.g., at the grain boundaries) of grains adjacent to plastically deformed regions, which hinder elastic relaxation. This leads to a change in the intergranular stress state between the uncut parent disk and the extracted reference sample. ${ }^{22-24}$ Since the intergranular stresses are not canceled out using the reference sample for residual stress analysis, intergranular stresses are interpreted as macroscopic stresses - also termed as spurious stress.

The level of spurious stresses changes through the thickness of the disk as they are governed by the elastic and/or plastic anisotropy of individual grains. Also, variations in the degree of pre-deformation, e.g., due to forging, across the disk thickness result in a non-uniform intergranular stress distribution: The grains in highly stressed regions may have already yielded, whereas other regions contain mainly elastically strained grains influencing the (elastic or plastic) mechanical behavior during macroscopic relaxation. Also, different local stress gradients in the initial stress state of the parent disk may transfer unequal loads to the surrounding material areas during unloading. This will lead to an increase of the non-uniformity of the level of spurious stresses.

A quantitative approach to derive the changes in intergranular stresses due to the cutting is to replace the measured reference values by calculated reference values. As suggested by the ISO standard, boundary conditions given by the mechanical equilibrium were used for the calculation. In 
the case of the disk, the mechanical equilibrium requires vanishing stresses in $x_{3}$-direction (see Sec. VI). Combining this with Hooke's law (Eq. (3)) one can calculate the required reference values from

$$
S_{0, \text { calc }}=\left(\frac{1-2 \nu_{h k l}}{1+\nu_{h k l}}\right) \cdot\left(S_{3}+\frac{\nu_{h k l}}{1-2 \nu_{h k l}} \cdot\left(S_{1}+S_{2}+S_{3}\right)\right)
$$

with $S_{0, \text { calc }}=1 / \sin \theta_{0, \text { calc }}, S_{i}=1 / \sin \theta_{x_{i}}$, and $i=1,2,3$, where $\theta_{x_{i}}$ are the scattering angles measured from the original disk $(R=50 \mathrm{~mm})$ in the principal direction $i$. Whereas the reference values are assumed to be equal in all three principal strain directions, they vary with the location along the measurement path. The distribution of these calculated reference values through the disk thickness is a measure of the variation of the stress free lattice spacing $d_{0}$. Varying lattice spacings are mainly caused by differences in the chemical composition and in the level of plastic pre-deformation through the sample. Assuming equal intergranular residual stress states in the original disk and in the samples with reduced radii, the calculated reference values must be valid for all sample radii.

Figure 5 shows the residual stress distribution of the sample with a radius of $R=2 \mathrm{~mm}$ determined on the basis of reference values calculated by Eq. (4). As the sample with a radius of $R=2 \mathrm{~mm}$ possesses dimensions small enough to be macroscopically stress-free, the use of appropriate reference values should give vanishing stresses in all 3 principal directions. The non-vanishing stresses in the $x_{1} / x_{2}$ as well as in the $x_{3}$ direction are indicative for differences in the intergranular residual stress states relative to the parent disk. Hence, the stresses shown in Figure 5 are a quantitative measure of the change of intergranular residual stresses during the extraction of the reference sample.

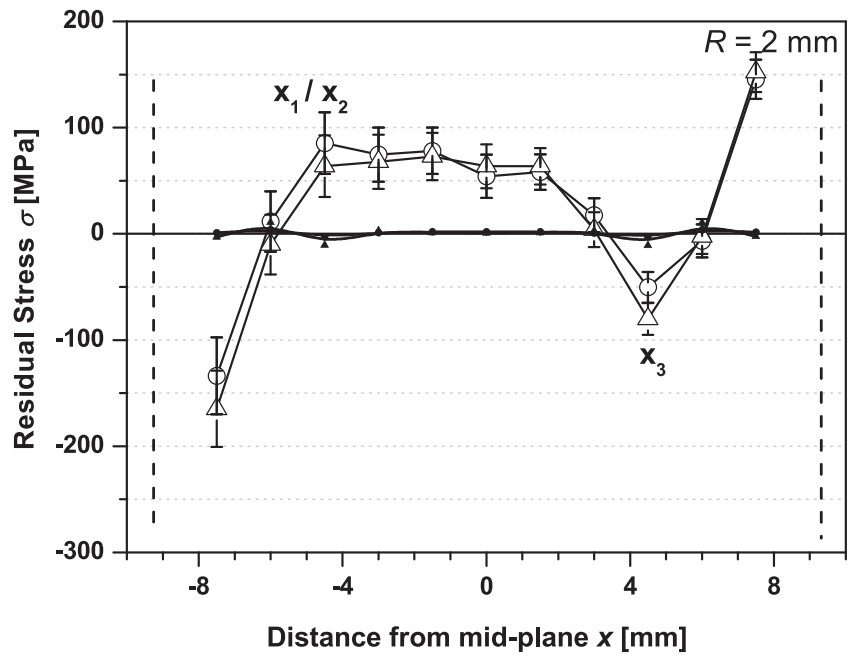

FIG. 5. Residual stress distributions of the sample with a radius of $R=2 \mathrm{~mm}$ as determined with neutron diffraction data on the basis of reference values, which were calculated incorporating the mechanical equilibrium for the original disk $(R=50 \mathrm{~mm})$. The stress distributions in $x_{1} / x_{2}$-directions (circles) and in $x_{3}$-direction (triangles) are given. Note: Only one of the two radial principal directions $\left(x_{1} / x_{2}\right)$ was measured due to the small dimensions of the sample. The macroscopic stresses predicted by the FE-model (filled symbols) are shown in addition to the experimental results (open symbols). The dashed lines on both sides of the graphs mark the upper and bottom surfaces of the disk.
Figure 6 shows the macroscopic residual stress distributions of the disks with radii of 50,20, and $10 \mathrm{~mm}$ determined on the basis of the reference values $\theta_{0, \text { calc, }}$, which were calculated

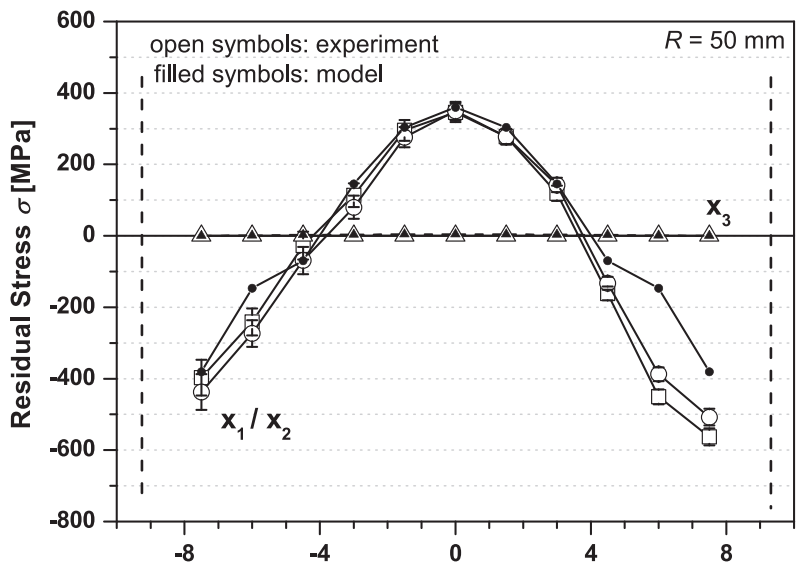

(a) Distance from mid-plane $x[\mathrm{~mm}]$
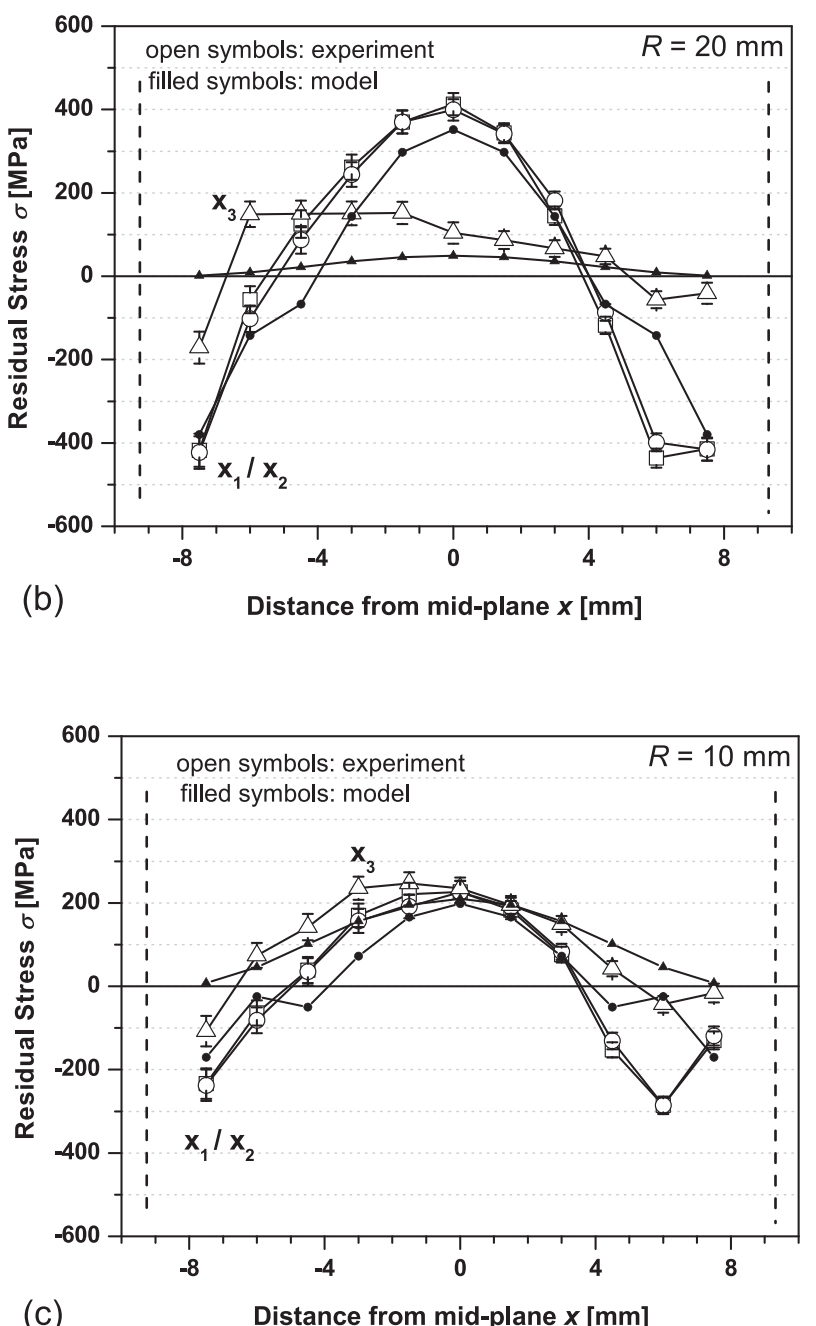

FIG. 6. Residual stress distributions of the samples with radii of (a) 50, (b) 20 , and (c) $10 \mathrm{~mm}$ along the principal axes $x_{1}$ (squares), $x_{2}$ (circles), and $x_{3}$ (triangles). The macroscopic stresses predicted by the FE-model (filled symbols) are shown in addition to the experimental results (open symbols). The dashed lines on both sides of the graphs mark the upper and bottom surfaces of the disk. 
using Eq. (4). The corresponding results of the finite element simulation are in good agreement with the stresses determined by neutron diffraction. Again, the stresses in $x_{1}$ and $x_{2}$ directions for each individual sample radius have to be similar, due to the measurement positions along the centeraxis and the radial symmetry of the disks. This is observed for experimental and simulated residual stress distributions. The simulated residual stresses in the axial direction show the effect of free edges on the macroscopic residual stress states: The axial stresses are not necessarily vanishing for all measurement points through the thickness for the disks with radii $<50 \mathrm{~mm}$ (compare Sec. VI). The remaining small asymmetries across the thickness of the disk in the experimentally determined stress distributions are a consequence of the high deformation rates applied during the forging process, which may lead to different deformation trajectories for the axial $\left(x_{3}\right)$ direction and the in-plane $\left(x_{1} / x_{2}\right)$ directions, leading to little differences in the reference values for these directions. These differences, however, are small and do not affect the presented results.

\section{CONCLUSION}

In residual stress analysis by neutron diffraction, small pieces of the strained component of interest often are cut and used as macrostress free reference samples for the determination of stress levels. This procedure ideally leads to identical intergranular stress states in reference and strained sample, thus microstresses will not play a role in the macroscopic stress analysis. However, especially for samples consisting of materials, which possess strong crystal anisotropy, combined with a large degree of plastic deformation, the intergranular stress state may change due to relaxation during extraction of reference samples. If this is not taken into account, the consequences will be that the analysis procedure yields significant spurious stresses, which are non-uniform over the sample dependent on its deformation history. In such a case, determination of absolute macroscopic stresses will be difficult by using measured reference values. It is therefore recommended that mechanical equilibrium conditions are used to calculate proper reference values if possible.

The elastic and plastic anisotropies of the material play an important role in the formation of intergranular stresses. Consequently, it is assumed that materials with less crystal anisotropy than nickel (e.g., aluminum) show smaller deviations in intergranular stresses and strains, whereas materials with large crystal anisotropy (e.g., steel) are expected to show larger changes in the intergranular stress state in similar conditions. The results presented here provide evidence that even lattice planes are affected, which are normally known for their low accumulation of intergranular stresses (e.g., $\{311\}$ in fcc nickel). It is likely that this effect is even more significant for other lattice planes (e.g., $\{220\}$ in nickel). This has to be verified by more experiments. These experiments should include time-of-flight measurements of this phenomenon, since here all Bragg reflections are measured simultaneously on the same sample. This allows to exclude different local microstructures when comparing the behavior of different Bragg reflections. This is not the case for constant wavelength diffractometer setups, where one has to use several samples—one for each Bragg reflection.

\section{ACKNOWLEDGMENTS}

We gratefully acknowledge the Deutsche Forschungsgemeinschaft $D F G$ for funding this research within Projects WE 2351/11-1 and PE 580/7-1. In addition, the authors thank the German neutron reactor FRM II for providing beam time at instruments STRESS-SPEC and SPODI. The authors want to acknowledge the instrument scientists of SPODI, M. Hölzel and A. Senyshyn, for their support during and after the powder diffraction measurement.

${ }^{1}$ M. T. Hutchings, P. J. Withers, T. M. Holden, and T. Lorentzen, Introduction to the Characterization of Residual Stress by Neutron Diffraction (Taylor \& Francis, Boca Raton, 2005).

${ }^{2}$ D. S. Kupperman, Annu. Rev. Mater. Sci. 24, 265-291 (1994).

${ }^{3}$ V. Hauk, Structural and Residual Stress Analysis by Nondestructive Methods (Elsevier Science B.V., Amsterdam, 1997).

${ }^{4}$ P. J. Withers and H. K. D. H. Bhadeshia, Mater. Sci. Technol. 17, 355-365 (2001).

${ }^{5}$ ISO/TS 21432:2005(E), Non-Destructive Testing-Standard Test Method for Determining Residual Stresses by Neutron Diffraction, 2005-07-15 (International Organization for Standardization, Geneva, Switzerland).

${ }^{6} \mathrm{R}$. A. Winholtz, in Analysis of Residual Stress by Diffraction using Neutron and Synchrotron Radiation, edited by M. E. Fitzpatrick and A. Lodini (Taylor \& Francis, London, 2003), pp. 60-77.

${ }^{7}$ B. Ortner, T. Antretter, M. Hofmann, and E. Werner, Mater. Sci. Forum 571-572, 225-229 (2008).

${ }^{8}$ Analysis of Residual Stress by Diffraction using Neutron and Synchrotron Radiation, edited by M. E. Fitzpatrick and A. Lodini (Taylor \& Francis, London, 2003).

${ }^{9}$ D. F. Paulonis and J. J. Schirra, in Superalloys 718, 625, 706 and Various Derivatives, edited by E. A. Loria (TMS, Warrendale, 2001), pp. 13-23.

${ }^{10}$ A. LeBail, H. Duroy, and J. L. Fourquet, Mater. Res. Bull. 23, 447-452 (1988).

${ }^{11}$ M. Hoelzel, A. Senyshyn, R. Gilles, H. Boysen, and H. Fuess, Neutron News 18(4), 23-26 (2007).

${ }^{12}$ J. Repper, M. Hofmann, C. Krempaszky, W. Petry, and E. Werner, Mater. Sci. Forum 571-572, 39-44 (2008).

${ }^{13}$ J. S. Robinson, D. A. Tanner, C. E. Truman, A. M. Paradowska, and R. C. Wimpory, Mater. Charact. 65, 73-85 (2012).

${ }^{14}$ M. Hofmann, J. Rebelo Kornmeier, U. Garbe, R. C. Wimpory, J. Repper, G. A. Seidl, H. G. Brokmeier, and R. Schneider, Neutron News 18(4), 2730 (2007).

${ }^{15}$ T. Manns and B. Scholtes, HTM J. Heat Treat. Mater. 65, 75-84 (2010).

${ }^{16}$ T. M. Holden, C. N. Tomé, and R. A. Holt, "Experimental and theoretical studies of the superposition of intergranular and macroscopic strains in Ni-based industrial alloys," Metall. Mater. Trans. A 29, 2967-2973 (1998).

${ }^{17}$ T. M. Holden, R. A. Holt, and A. P. Clarke, "Intergranular strains in Inconel-600 and the impact on interpreting stress fields in bent steamgenerator tubing," Mater. Sci. Eng. A 246, 180-198 (1998).

${ }^{18}$ K. Kolarik, N. Ganev, Zd. Pala, J. Barcl, T. Bakalova, and M. Svanter, MM Sci. J. 2009(4), 159-161.

${ }^{19}$ B. Ekmekci, Appl. Surf. Sci. 253, 9234-9240 (2007).

${ }^{20}$ C. Krempaszky, E. Werner, M. Stockinger, in Superalloys 718, 625, 706 and Derivatives, edited by E. A. Loria (TMS, Warrendale, 2006), pp. 527538.

${ }^{21}$ A. J. Fletcher and C. Lewis, Mater. Sci. Technol. 1(10), 780-785 (1985).

${ }^{22}$ D. Dye, H. J. Stone, and R. C. Reed, Curr. Opin. Solid State Mater. Sci. 5, 31-37 (2001).

${ }^{23}$ H. J. Stone, T. M. Holden, and R. C. Reed, Acta Mater. 47(17), 44354448 (1999).

${ }^{24}$ B. Clausen, T. Lorentzen, and T. Leffers, Acta Mater. 46(9), 3087-3098 (1998). 